ACCEPTED MANUSCRIPT

\title{
Ising pairing in atomically thin superconductors
}

To cite this article before publication: Ding Zhang et al 2021 Nanotechnology in press https://doi.org/10.1088/1361-6528/ac238d

\section{Manuscript version: Accepted Manuscript}

Accepted Manuscript is "the version of the article accepted for publication including all changes made as a result of the peer review process, and which may also include the addition to the article by IOP Publishing of a header, an article ID, a cover sheet and/or an 'Accepted Manuscript' watermark, but excluding any other editing, typesetting or other changes made by IOP Publishing and/or its licensors"

This Accepted Manuscript is @ 2021 IOP Publishing Ltd.

During the embargo period (the 12 month period from the publication of the Version of Record of this article), the Accepted Manuscript is fully protected by copyright and cannot be reused or reposted elsewhere.

As the Version of Record of this article is going to be / has been published on a subscription basis, this Accepted Manuscript is available for reuse under a CC BY-NC-ND 3.0 licence after the 12 month embargo period.

After the embargo period, everyone is permitted to use copy and redistribute this article for non-commercial purposes only, provided that they adhere to all the terms of the licence https://creativecommons.org/licences/by-nc-nd/3.0

Although reasonable endeavours have been taken to obtain all necessary permissions from third parties to include their copyrighted content within this article, their full citation and copyright line may not be present in this Accepted Manuscript version. Before using any content from this article, please refer to the Version of Record on IOPscience once published for full citation and copyright details, as permissions will likely be required. All third party content is fully copyright protected, unless specifically stated otherwise in the figure caption in the Version of Record.

View the article online for updates and enhancements. 


\title{
Ising pairing in atomically thin superconductors
}

\author{
Ding Zhang ${ }^{1,2,3,4, *}$ and Joseph Falson ${ }^{5,6, \dagger}$ \\ ${ }^{1}$ State Key Laboratory of Low Dimensional Quantum Physics and Department of Physics, \\ Tsinghua University, Beijing 100084, China. \\ ${ }^{2}$ RIKEN Center for Emergent Matter Science (CEMS), Wako, Saitama 351-0198, Japan. \\ ${ }^{3}$ Beijing Academy of Quantum Information Sciences, Beijing 100193, China. \\ ${ }^{4}$ Frontier Science Center for Quantum Information, Beijing 100084, China. \\ ${ }^{5}$ Department of Applied Physics and Materials Science, \\ California Institute of Technology, Pasadena, CA, USA. \\ ${ }^{6}$ Institute for Quantum Information and Matter, \\ California Institute of Technology, Pasadena, CA, 91125, USA
}

(Dated: August 28, 2021)

\begin{abstract}
Ising-type pairing in atomically thin superconducting materials has emerged as a novel means of generating devices with resilience to a magnetic field applied parallel to the two-dimensional plane. In this mini-review, we canvas the state of the field by giving a historical account of two-dimensional superconductors with strongly enhanced in-plane upper critical fields, together with the type-I and type-II Ising pairing mechanisms. We highlight the vital role of spin-orbit coupling in these superconductors and discuss other effects such as symmetry breaking, atomic thicknesses, etc. Finally, we summarize the recent theoretical proposals and highlight the open questions, such as exploring topological superconductivity in these systems and looking for more materials with Ising pairing.
\end{abstract}

*dingzhang@mail.tsinghua.edu.cn

† falson@caltech.edu 


\section{INTRODUCTION}

Two years after his discovery of superconductivity, Kamerlingh Onnes envisioned that superconductors could be used for generating strong magnetic fields on the order of tens of Tesla [1]. It soon turned out that superconductors discovered in the earlier times, so-called type-I superconductors, easily lost their dissipationless property in a magnetic field with a field strength as low as tens of millitesla. It was until the discovery of type-II superconductors that Onnes' initial dream became practical. Today, scientists around the world use such superconductors to generate high magnetic fields on a daily basis in medical and research settings. The field strengths of superconducting magnets stay on the order of tens of Tesla, which are governed by a fundamental parameter of the superconductor-the upper critical field $\left(B_{c 2}\right)$. For type-I superconductors, the magnetic field either gets fully screened or penetrates through the whole compound and destroys superconductivity. A first order transition occurs at a critical field of $B_{c}$. For type-II superconductors, however, the magnetic field lines can be first trapped in a bundle of pinholes-vortices with quantized fluxes, leaving the rest of the bulk still superconducting. The onset of such a mixed state is marked by the lower critical field- $B_{c 1}$. It is until the density of the vortices, which increases with the magnetic field, reaches a certain threshold that the superconducting state and the normal state become energetically indistinguishable. At this density with a corresponding magnetic field- $B_{c 2}$, the superconductor returns to the normal state.

One could lift the upper bound imposed by the vortex effect by drastically reducing the crosssection of a superconductor in the magnetic field. In other words, a thin film superconductor can be much robust against an in-plane magnetic field. The Ginzburg-Landau theory predicts that such an in-plane upper critical field- $B_{c 2, \|}$ increases rapidly with the reduction of film thicknesses [2]. In ultrathin superconductors, however, the orbital effect brought by vortices becomes negligible. Instead, the spin paramagnetism emerges as the main mechanism that brings the superconductor with singlet pairing back to the normal state. A sufficiently strong magnetic field can polarize all electronic spins such that Cooper pairs with anti-aligned spins break up [3, 4]. This magnetic field is the so-called Pauli limit: $B_{p}$. By equating the superconducting binding energy with the paramagnetic energy of the normal state, one obtains that $B_{p}^{B C S}=1.86 T_{c 0}(\mathrm{~T})$, where $T_{c 0}$ is the transition temperature. We add BCS in our notation because the formula above is obtained by assuming a standard BCS ratio of $2 \Delta=3.53 k_{B} T_{c 0}$ for weak superconductors and a $g$-factor of 2 . These assumptions are not necessarily followed in realistic situations. For example, the gap to $T_{c 0}$ 
ratio may be much larger than the BCS ratio [5]. On the other hand, the $g$-factor can differ from 2 , as pointed out in literatures $[6,7]$. The aforementioned effects may result in a value of $B_{p}$ that deviates from $B_{p}^{B C S}$. Here, however, we employ $B_{p}^{B C S}$ as a conventional tool to normalize $B_{c 2}$ such that $B_{c 2} / B_{p}^{B C S}$ serves as the figure-of-merit when different superconductors are compared.

In aluminum films, Tedrow and Meservey [2] demonstrated that the spin paramagnetic effect was largely obeyed, although the additional effect of spin-orbit scattering should have been taken into account. Two 5-nm thick aluminum samples, for example, possessed slightly enhanced $T_{c 0}$ of 2.04 and $2.15 \mathrm{~K}$, in comparison to the bulk value of $1.2 \mathrm{~K}$. Their corresponding $B_{c 2, \|}$ were 4.01 and $4.05 \mathrm{~T}$, close to the calculated $B_{p}^{B C S}$ of 3.79 and $4.00 \mathrm{~T}$ (One exemplary data set digitized from the original paper is shown in Fig. 2a). Furthermore, the aluminum thin films hosted the predicted first-order transition to the normal state at low temperature, due to the spin-paramagetic effect.

Since it was derived based on a simple model regarding the electronic spin, the Pauli limit can be surpassed in situations where the spin orientation departs from the ideal isotropic case. In superconducting thin films incorporated with heavy elements, spin-orbit scattering can randomize the spins and substantially weaken the polarizing effect of the magnetic field [8]. Thin films may also experience the breaking of inversion symmetry along the normal direction from the substrate to the top surface. It leads to the Rashba effect that tends to align the spins in different directions in the plane, which can essentially enhance $B_{c 2}$ up to $\sqrt{2} B_{p}^{B C S}$ [9]. In clean superconductors, above $B_{p}^{B C S}$, the uniformly superconducting state can get replaced by a spatially ordered state -FuldeFerrell-Larkin-Ovchinnikov (FFLO) state with partial spin polarizations [10, 11]. Another exotic scenario is spin triplet pairing where the system would not be affected by the Pauli paramagnetic effect at all.

By the end of 2015, it became clear that $B_{c 2, \|}$ in the atomically thin highly crystalline superconductors [12-14] can greatly surpass $B_{p}^{B C S}$. Yet none of the aforementioned mechanisms were able to explain this enhancement. It marked the advent of so-called Ising superconductors. To date, the Ising superconductivity had been discovered in many transition metal dichalcogenides $[6,15-$ 18], as shown in Fig. 1. Their robustness of superconductivity against the in-plane magnetic field originates from the strong spin-orbit coupling together with the breaking of in-plane inversion symmetry, as was considered earlier for non-centrosymmetric superconductors of bulk crystals $[19,20]$. Lately, enhanced $B_{c 2}$ in atomically thin superconductors without breaking in-plane inversion symmetry has also been reported [21, 22] (Fig. 1). It suggests a distinct type of Ising pairing in materials with strong spin-orbit couplings hosting multi-degeneracies at high symmetry 
points $[23,24]$. Further theoretical work not only outlines a broader material pool of potential Ising superconductors but also suggests a possible realization of topological superconductivity in these systems [25-39].

This mini-review aims at providing a progress report of atomically thin superconductors with enhanced $B_{c 2}$ that are mostly attributed to Ising pairing (Fig. 1). Reviews on a broader scope or some other specific aspects of two-dimensional (2D) superconductors can be found in Refs [4044]. Here we collect data points from the reported Ising superconductors, hoping to construct a unique perspective at the enhancement of $B_{c 2}$. We discuss the influence of spin-orbit coupling strengths, sample thicknesses, etc. while ending with some open questions in the field.

\section{DISCOVERY OF ISING SUPERCONDUCTORS}

Thanks to technical advancements, there are now a number of viable pathways to creating ultrathin crystals that can show superconductivity [40,42]. One direction is to directly synthesize thin films of a material, for example through molecular beam epitaxy (MBE). Another is through mechanical exfoliation where sheets of van der Waals materials can be isolated down to a few atomic layers. Alternatively, it is possible to study the physics of thin layers by selectively accumulating carriers on the surface of bulk crystals, for example with ionic liquid (IL) gating. The enhanced $B_{c 2, \|}$ has become one intriguing property of these 2D superconductors. Here we give a historical account of selected 2D superconductors with enhanced $B_{c 2, \|}$. Figure 1 acts to guide our discussion by plotting the ratio of $B_{c 2, \|} / B_{p}^{B C S}$ from reports in the literature according to their publication date.

In a 2012 systematic study of $\delta$-doped $\mathrm{SrTiO}_{3}$ heterostructures, Kim et al. [45] noted that $B_{c 2, \|}(T \rightarrow 0)$ exceeded $B_{p}^{B C S}$ by a factor of 4 . We show one exemplary data set digitized from the original paper in Fig. 2e. An earlier study on $\mathrm{LaAlO}_{3} / \mathrm{SrTiO}_{3}$ interface superconductors [46] already found a large $B_{c 2, \|} / B_{p}^{B C S}$ ratio of 3.5. The $\delta$-doped films embedded between cap and buffer layers of $\mathrm{SrTiO}_{3}$ has the advantage due to its symmetric structure. It helps exclude the Rashba effect as the mechanism for the enhancement. For a quantitative analysis, Kim et al. [45] employed the Werthamer-Helfand-Hohenberg (WHH) formula:

$$
\begin{aligned}
\ln \left(\frac{T_{c 0}}{T}\right) & =\left(\frac{1}{2}-\frac{i \lambda_{\mathrm{SO}}}{4 \gamma}\right) \psi\left(\frac{1}{2}+\frac{\bar{h}+\frac{1}{2} \lambda_{\mathrm{SO}}-i \gamma}{2 T / T_{c 0}}\right) \\
& +\left(\frac{1}{2}+\frac{i \lambda_{\mathrm{SO}}}{4 \gamma}\right) \psi\left(\frac{1}{2}+\frac{\bar{h}+\frac{1}{2} \lambda_{\mathrm{SO}}+i \gamma}{2 T / T_{c 0}}\right)-\psi\left(\frac{1}{2}\right),
\end{aligned}
$$


where $\psi$ is the digamma function and the rest of the terms are:

$$
\begin{gathered}
\bar{h}=\frac{e v_{F}^{2} \tau_{\mathrm{T}} B}{3 \pi k_{B} T_{c 0}}, \\
\lambda_{\mathrm{SO}}=\frac{2 \hbar}{3 \pi k_{B} T_{c 0} \tau_{\mathrm{SO}}}, \\
\gamma=\sqrt{\left(\frac{\mu_{B} B}{\pi k_{B} T_{c 0}}\right)^{2}-\frac{1}{4} \lambda_{\mathrm{SO}}^{2},}
\end{gathered}
$$

where $v_{F}$ is the Fermi velocity, $\tau_{\mathrm{T}} / \tau_{\mathrm{SO}}$ the transport/spin-orbit scattering time, $\mu_{B}$ the Bohr magneton. With the sample getting thinner, they found an unusual decrease of the extracted $\tau_{\text {SO }}$ while $\tau_{\mathrm{T}}$ stayed almost unchanged. This dichotomy suggested that the intrinsic spin-orbit coupling may play a role in giving rise to the large $B_{c 2, \|}(T \rightarrow 0) / B_{p}^{B C S}$ ratio.

Indications for the intrinsic effect of spin-orbit coupling were also found in 2013. Sekihara, Masutomi and Okamoto studied submonolayer of $\mathrm{Pb}$ films grown on GaAs(110) substrate [47] (Fig. 2e). There, the superconducting transition temperature dropped by as little as $2 \%$ in the in-plane magnetic field up to $14 \mathrm{~T}$. The following equation was employed to fit the data:

$$
\ln \left(\frac{T_{c 0}}{T}\right)=\psi\left(\frac{1}{2}+\frac{3 \tau_{\mathrm{SO}}}{2 \hbar} \frac{\left(\mu_{B} B\right)^{2}}{2 \pi k_{B} T}\right)-\psi\left(\frac{1}{2}\right) .
$$

This equation is usually refereed to as the Klemm-Luther-Beasley (KLB) formula [8]. It can be derived from Eq. (1) when considering strong spin-orbit scattering for superconductors in the dirty limit, i.e. $\lambda_{\text {SO }} \rightarrow \infty$ and $\tau_{\mathrm{T}}<<\tau_{\text {SO }}$. The extracted $\tau_{\text {SO }}$ was found to be comparable with the estimated $\tau_{\mathrm{T}}$ of the Pb films: $\tau_{\mathrm{T}} \sim 0.8 \tau_{\mathrm{SO}}$. This is unusual because $\tau_{\mathrm{SO}}$ should be orders of magnitude larger than $\tau_{\mathrm{T}}$. The authors therefore proposed that the intrinsic Rashba spin splitting in their system may give rise to an inhomogeneous superconducting state similar to the FFLO state, which might account for the enhanced $B_{c 2, \|}$. A similar conclusion was arrived by Nam et al. [48] when studying their five-monolayer $\mathrm{Pb}$ films grown on $\mathrm{Si}(111)$ substrates. The researchers suggested that the orbital effect, which is often neglected when considering purely a 2D superconductor, may be still responsible for pair-breaking because the spin degree of freedom is quenched. It highlights the importance of a close inspection of theoretical expectation of $B_{c 2, \mid,}$ from different mechanisms.

The breakthrough of identifying a novel mechanism different from Rashba spin splitting or spin-orbit scattering happened in 2015/16. Three individual groups observed strongly enhanced $B_{c 2, \mid]}$ in their atomically thin superconductors of $2 \mathrm{H}-\mathrm{MoS}_{2}$ (Ref.[12 and 13]) or $2 \mathrm{H}-\mathrm{NbSe}{ }_{2}$ (Ref.[14]) (Fig. 2a). Experimentally, the researchers obtained atomically thin crystalline superconductors via two approaches. For thin flakes of $2 \mathrm{H}-\mathrm{MoS}_{2}$, Lu et al. [12] and Saito et al. [13] 
employed the ionic liquid gating technique to electrostatically inject charge carriers and tune the top one to two layers into the superconducting state. On the other hand, Xi et al. [14] were able to mechanically exfoliate $2 \mathrm{H}-\mathrm{NbSe}_{2}$ down to a monolayer and still preserve the superconductivity. They found that $B_{c 2, \|}$ exceeded $B_{p}^{B C S}$ by a factor of 4 to 6 . Notably, the extracted $\tau_{\text {SO }}$ by using Eq. (5) to fit the data became shorter than $\tau_{\mathrm{T}}$. This is unreasonable because the transport scattering time should be the shortest. By ruling out the spin-orbit scattering mechanism, the researchers proposed an intrinsic mechanism. It arises from the strong spin-orbit coupling together/with the lack of in-plane inversion symmetry in the individual atomic layers of $\mathrm{MoS}_{2}$ and $\mathrm{NbSe}_{2}$. Taking a monolayer of $\mathrm{MoS}_{2}$-one S-Mo-S sequence-for an example, its band structure consists of electron pockets located around the $K$ and $K^{\prime}$ points of the Brillouin zone Strong spin-orbit coupling induces spin splitting of the bands. Due to the broken inversion symmetry of its crystal structure, the spin splitting has opposite signs at the $K$ and $K^{\prime}$ points. In the superconducting state, Cooper pairs are built up by the electrons from the two valleys. Their spins are locked to the out-of-plane direction due to the rotational symmetry. This is schematically shown in Fig. 1. An in-plane magnetic field therefore has to compete with the Zeeman-like spin splitting built into the bands, thus the superconducting state becomes more resilient to the paramagnetic effect. Such a mechanism in $2 \mathrm{D}$ superconductors is called Ising superconductivity.

In fact, an enhanced $B_{c 2, \|}$ over the Pauli limit was proposed earlier in bulk crystals without symmetry centers-noncentrosymmetric superconductors by Bulaevskii, Guseinov, and Rusinov [19] as well as by Frigeri et al. [20]. By employing the specific Hamiltonian suitable for transition metal dichalcogenide monolayers, Ilic, Meyer and Houzet [49] derived the following formula for the temperature dependence of the upper critical field for Ising superconductivity:

$$
\ln \left(\frac{T_{c 0}}{T}\right)=\frac{\mu_{B}^{2} B^{2}}{\beta_{\mathrm{SO}}^{* 2}+\mu_{B}^{2} B^{2}} \operatorname{Re}\left[\psi\left(\frac{1}{2}+\frac{i \sqrt{\beta_{\mathrm{SO}}^{* 2}+\mu_{B}^{2} B^{2}}}{2 \pi k_{B} T}\right)-\psi\left(\frac{1}{2}\right)\right],
$$

where $\beta_{\text {SO }}^{*}$ is the effective spin-orbit coupling strength, which may be renormalized from the intrinsic spin-orbit coupling by the disorder scattering [5].

The concept of Ising superconductivity was further bolstered by the subsequent studies of other transition metal dichalcogenides monolayers. In a $\mathrm{WS}_{2}$ monolayer, superconductivity was again realized by the ionic liquid gating technique [15]. There, for the superconducting state with $T_{c 0}=$ $1.54 \mathrm{~K}$, Lu et al. found that the transition temperature only decreased by $5 \%$ when a strong in-plane magnetic field of $35 \mathrm{~T}$ was applied [15](Fig. 2b). In a monolayer exfoliated from $2 \mathrm{H}-\mathrm{TaS}_{2}$ crystals, 
de la Barrera et al. found that $B_{c 2,||}$ exceeded the highest magnetic field of their magnet (34.5 T) when the temperature was below $60 \%$ of $T_{c 0}$ [16] (Fig. 2b). They further carried out a systematic study by comparing the enhanced $B_{c 2, \|}$ in both $\mathrm{TaS}_{2}$ and $\mathrm{NbSe}_{2}$. The larger $B_{c 2, \|} / B_{p}^{B C S}$ ratio in $\mathrm{TaS}_{2}$ originated from its stronger spin-orbit coupling strength, which was consistent with the density functional theory calculations. Enhanced $B_{c 2, \|}$ was also found in monolayer $\mathrm{WTe}_{2}[50,51]$ as well as few-layers of $1 \mathrm{~T}_{d}-\mathrm{MoTe}_{2}$ [52] (Fig. 2f). A variant of Ising superconductivity was proposed that considered the tilted spins in the momentum space. Recently, Rhodes et al. [53] further observed enhanced $B_{c 2, \|}$ in a monolayer of $\mathrm{MoTe}_{2}$. These materials suggest a broader scope for locked spins in superconductors with strong spin-orbit couplings [54].

We remark that there exist two different approaches for estimating the spin-orbit coupling strength for Ising pairing. Some studies employed Eq. (5) for fitting their data but interpret $3 \hbar / 2 \tau_{S O}$ as $\beta_{S O}[14,16]$. Others used the formula for Ising superconductivity-similar to Eq. (6)to extract $\beta_{S O}^{*}[12,15]$. Due to the completely different origins, the fitted values can greatly differ. For example, applying Eq. (5) directly to the data of $\mathrm{MoS}_{2}$ (orange curve in Fig. 2a) gives rise to $3 \hbar / 2 \tau_{S O}=18 \mathrm{meV}$. By contrast, the best fit to the same data set by using Eq. (6) yields $\beta_{S O}^{*}=3.4 \mathrm{meV}$. This value is slightly smaller than the reported value of $6 \mathrm{meV}$ in literature [12]. This difference stems from the fact that Eq. (6) does not take into account the Rashba effect. Another example is $\mathrm{NbSe}_{2}$. Applying Eq. (6) to the data set in Fig. 2 a gives rise to $\beta_{S O}^{*}=4.1 \mathrm{meV}$, which is much smaller than the reported value: $2 \beta_{S O}=76 \mathrm{meV}$ [14] when Eq. (5) was employed. One should therefore be cautious in drawing comparison between the spin-orbit coupling strengths based on the listed values in the literatures.

Figure 3 compares attributes of transition metal dichalcogenide superconductors based upon their layer number and chemical composition. We draw upon two key parameters; the thickness of samples studied and the cation size. While the data set is incomplete, a trend is visible in both graphs. The $B_{c 2, \|} / B_{p}^{B C S}$ ratio is enhanced by both thinning the sample (Fig. 3a) and enhancing the cation size (Fig. 3b). Thinning the sample, preferably to the monolayer limit, is necessary to realize the inversion symmetry breaking of the lattice. It is nevertheless important to note that the enhancement in the upper critical field persists even in multi-layered $\mathrm{NbSe}_{2}$ and $\mathrm{TaS}_{2}$. It indicates that the local inversion symmetry breaking in the plane is sufficient for spin-orbital locking, although the global inversion symmetry is not broken in multi-layer systems. The effect of cation is associated with the increase in spin-orbit coupling with its increasing $Z$ value, which enters the superconducting behavior in the form of $\beta_{\mathrm{SO}}^{*}$. The data in Fig. 3 also highlight that the enhanced 
$B_{c 2, \|}$ is a reproducible result. Three independent studies of $\mathrm{NbSe}_{2}[6,14,16]$ present similar values of $B_{c 2, \|} / B_{p}^{B C S}$ at the lowest measured temperatures, along with the value obtained at $T=0$ through a KLB fit. Notably, one of the studies explored the macroscopic $\mathrm{NbSe}_{2}$ sample grown by MBE [6]. The authors employed a pulsed magnet in combination with ultra-low temperature facility to experimentally determine $B_{c 2, \|} / B_{p}^{B C S}$ down below $0.15 T_{c 0}$. The large enhancement in MBE grown samples is of technical importance for further investigations of Ising pairing and potential applications.

Among the transition metal dichalcogenide superconductors, a missing feature of Ising superconductivity predicted by theory was the up-turn behavior of $B_{c 2, \|}$ at low temperature as described by Eq. (6). In monolayer cases of $\mathrm{NbSe}_{2}$ and $\mathrm{TaS}_{2}[14,16]$ as well as in the ionic liquid gated $\mathrm{WS}_{2}$ [15], the upper critical field quickly exceeded the highest magnetic field available when the temperature is still close to $T_{c 0}$, such that the behavior of $B_{c 2, \|}$ at $T \rightarrow 0$ was unattainable. For bilayer $\mathrm{NbSe}_{2}$, trilayer $\mathrm{TaS}_{2}$, as well as ionic liquid gated $\mathrm{MoS}_{2}$ [13], on the other hand, $B_{c 2, \|}$ showed a saturating behavior at $T / T_{c 0}<0.5$. It was suggested that the Rashba effect $[12,13]$ and intervalley scattering [49] in these films can renormalize the temperature behavior so that the up-turn at low temperature gets smeared out.

The non-saturating behavior was first found in a two-dimensional superconductor outside the transition metal dichalcogenide family. This time, samples grown by MBE became the protagonist. Liu et al. measured thin films of $\mathrm{Pb}$ grown on a special surface reconstruction layer of $\mathrm{Pb}$-striped incommensurate phase-on $\mathrm{Si}(111)$ substrate. They observed pronounced enhancement of $B_{c 2, \|}$ [5]. There, a more realistic $B_{p}$ rather than $B_{p}^{B C S}$ was estimated based on the known gap to $T_{c}$ ratio of $\mathrm{Pb}$. Intriguingly, they observed that $B_{c 2,||}$ in the $\mathrm{Pb}$ films with a thickness of six monolayers kept increasing with decreasing temperature down to $T \sim 0.25 T_{c 0}$ (Fig. 2b). Although the crystal structure of $\mathrm{Pb}$ itself is centrosymmetric, the authors pointed out that their special substrate hosted lattice distortion with its symmetry breaking effect permeating to thin films grown on top. Together with the strong spin-orbit coupling, ultrathin $\mathrm{Pb}$ films therefore possessed Ising superconductivity.

In another elemental superconductor-Sn, Liao et al. [55] found that $B_{c 2, \|}$ could exceed $B_{p}^{B C S}$ by a factor of 3. Their superconductors were made of a few atomic layers of strained $\alpha$-Sn-socalled stanene-grown by $\mathrm{MBE}$ on $\mathrm{PbTe} / \mathrm{Bi}_{2} \mathrm{Te}_{3}$ epitaxial layers upon a silicon (111) substrate. By measuring few-layer stanene at even lower temperatures down to $0.02 T_{c}$, Falson et al. [21] were able to map out almost the complete phase diagram of $B_{c 2, \|}(T)$. The same sample, as studied in Ref. [55] actually hosted an ever-growing $B_{c 2, \|}$ as $T$ decreased such that $B_{c 2, \|} / B_{p}^{B C S}$ eventually 
exceeded 4 (Fig. 2c). Of particular interest, was the up-turn behavior at low temperature, which echoed the prediction for Ising superconductivity [Eq. (6)]. However, stanene is sharply different from $\mathrm{NbSe}_{2}$ or $\mathrm{MoS}_{2}$ : its crystal structure is centrosymmetric; the electronic bands are around the $\Gamma$ point, as found by angular resolved photoemission spectroscopy (ARPES). The criteria of the then established Ising superconductivity were not satisfied. A new theoretical framework was proposed to explain this distinct type of behavior-type-II Ising superconductivity [23, 24]. Their theory was applicable to centrosymmetric materials with multiple degenerate orbitals. Taking Sn for example, bands around the Fermi level stem from $p_{x}$ and $p_{y}$ orbitals. Without spin-orbit interactions, the orbitals have four-fold degeneracy at the $\Gamma$ point: $\left|p_{x}\right\rangle,\left|p_{y}\right\rangle$, spin-up $(|\uparrow\rangle)$ and spin-down $(|\downarrow\rangle)$. The spin-orbit coupling lifts one layer of degeneracy and gives rise to two sets of bands: $\left(\left|p_{x}+i p_{y}, \uparrow\right\rangle,\left|p_{x}-i p_{y}, \downarrow\right\rangle\right)$ and $\left(\left|p_{x}-i p_{y}, \uparrow\right\rangle,\left|p_{x}+i p_{y}, \downarrow\right\rangle\right)$. Here the effective Zeeman splitting occurs between $\left|p_{x} \pm i p_{y}, \uparrow\right\rangle$ and $\left|p_{x} \pm i p_{y}, \downarrow\right\rangle$. This spin-orbit locking effect produces essentially the same spin configurations required by Ising superconductivity, and is schematically shown in Fig. 1. Based on this argument, Falson et al. [21] demonstrated that their theoretical model could satisfactorily fit the experimental data. Moreover, the model also explained why the up-turn was not always prominent even for the same triple layer $\mathrm{Sn}$ but on $\mathrm{PbTe}$ with different thicknesses (Fig. 2c). It stemmed from the enhanced spin-tilting of the hole band as the Fermi momentum increased with thinner PbTe. To further confirm this scenario, it is desirable to carry out a more systematic study of the enhanced $B_{c 2, \|}$ as a function of the Fermi level. We further note that few-layer stanene constitutes a unique platform as properties close to $T / T_{c 0}=0$ can be addressed. Future experiments should be directed to the understanding of the transition from the purely paramagnetism dominated regime to the orbital effect dominated regime.

In fact, there exists not just one type-II Ising superconductor. In ultrathin $\mathrm{PdTe}_{2}$ grown by MBE, Liu et al. [22] observed largely enhanced $B_{c 2, \|}$, exceeding $B_{p}$ by a factor of 6-8 (Fig. 2d). Notably, the $\mathrm{PdTe}_{2}$ compound also has a centrosymmetric crystal structure and hosts electronic bands around $\Gamma$ point. Theoretical analysis pointed out that the enhanced $B_{c 2, \|}$ was caused by type-II Ising pairing. Notably, few-layer stanene and $\mathrm{PdTe}_{2}$ are quite robust against air-exposure. By contrast, ultrathin $\mathrm{NbSe}_{2}$ and $\mathrm{TaS}_{2}$ are quite fragile and require handling in glove-boxes and capping with protection layers. In retrospect, the enhanced in-plane upper critical field in liquid gated $\mathrm{SnSe}_{2}$ as well as $1 \mathrm{~T}^{\prime}-\mathrm{MoS}_{2}$ also might be accounted for by type-II Ising paired superconductivity $[23,56]$. Together, these recent findings highlighted a broader scope of 2D superconductors hosting largely enhanced $B_{c 2, \|}$ due to the strong spin-orbit coupling. 
Following the initial experimental discoveries, further theoretical analysis suggests more exotic physics lurking in Ising superconductors, with the in-plane magnetic field as a tuning parameter. In order to experimentally verify many of the predictions, it is important to obtain information beyond temperature and magnetic field dependent resistances. Attempts to reveal the density of states in Ising superconductors have provided fruitful results. Both Sohn et al.[57] and Dvir et al.[58] fabricated planar tunnel junctions on $\mathrm{NbSe}_{2}$ down to a trilayer. Either a few layers of $\mathrm{MoS}_{2}$ or a thin film of $\mathrm{AlO}_{x}$ were used as the barrier. On the other hand, Costanzo et al. [59] utilized the band bending in the liquid gated $\mathrm{MoS}_{2}$ on multilayer graphene to realize a superconductorinsulator-metal junction. All three groups found that the superconducting gap could be protected by spin locking and stayed almost constant at small in-plane magnetic fields below around 10 T. Intriguingly, as revealed by Sohn et al., the gap gets continuously suppressed by ramping up the in-plane magnetic field to about $38 \mathrm{~T}$, rather than a first-order phase transition expected for a BCS superconductor [57]. The continuous transition lends further support to the scenario of Ising superconductivity. Due to the strong spin-locking of the Ising superconductor, its in-plane spin susceptibility in the superconducting state stays comparable to that in the normal state. The free energy of the superconducting state therefore continuously connects to that of the normal state. Further exploring the potential of planar junctions, Kang et al. [60] fabricated a sandwich structure consisted of $\mathrm{NbSe}_{2} / \mathrm{CrBr}_{3} / \mathrm{NbSe}_{2}\left(\mathrm{CrBr}_{3}\right.$ is a ferromagnetic insulator). They observed nearly $100 \%$ tunneling anisotropic magnetoresistance.

\section{OUTLOOK}

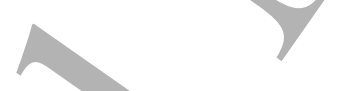

One enticing property of Ising superconductors is the predicted non-trivial topology. Indications of unconventional pairing were recently found in few-layer $\mathrm{NbSe}_{2}$, which manifested as two-fold anisotropy in the in-plane upper critical field [61]. In fact, possible $p$-wave pairing was predicted theoretically for a monolayer of $\mathrm{MoS}_{2}$ before the strongly enhanced $B_{c 2, \|}$ was experimentally observed [62]. A spin triplet $s$-wave was also proposed for certain on-site and nearest neighbor interaction strengths. Möckli and Khodas predicted that the in-plane magnetic field may induce nodes in the dispersion with Majorana flatbands connecting them, giving possibility of realizing nodal topological superconductivity [31]. Similar conclusion was arrived by He et al. [29]. By further taking into account the possible Rashba splitting in monolayer $\mathrm{NbSe}_{2}$, Shaffer et al. [35] predicted that the system may enter a topologically trivial phase unless the in-plane magnetic field 
is applied along one of $\Gamma-K$ lines, where a crystalline topological superconducting phase occurs. It was also proposed that nodal topological phases could be achieved in magnetic islands placed on Ising superconductors [36].

As already alluded to, triplet pairing is another intriguing property that may accompany Ising superconductivity. Möckli and Khodas [31,32] suggested that the superconducting state in monolayer TMD becomes parity mixed with triplet pairing-particularly the $f$-wave. The mixing of singlet and triplet pairing in monolayer $\mathrm{NbSe}_{2}$ was also confirmed by Wickramaratne et al. [34] through density functional theory (DFT) calculations. This triplet pairing may account for the further enhancement of $B_{c 2, \|}$ and can still persist when disorder effect is taken into account [33]. It can also affect the density of states [63]. A recent experimental study on the superconducting gap has indeed unveiled indication of such triplet pairing [64].

However, the triplet pairing may get masked by the dominant singlet pairing. Several theoretical groups thus outlined ways to extract the triplet pairing component. Zhou et al. [25] proposed that the triplet pairing in Ising superconductors can induce Majorana fermions in a semi-metal wire via proximity effect. The configuration is similar to the case of nano-wires with strong Rashba splittings that are proximitized by conventional $s$-wave superconductors [65]. However, the spin directions for the proposed semi-metal wire on a Ising superconductor lie in-plane instead of out-of-plane. The induced superconductivity was further proven to be robust against the inplane Zeeman fields [66]. In a similar spirit, spin triplet Andreev reflection was proposed to occur between a ferromagnet-Ising superconductor junction [30]. An in-plane junction made of Ising superconductor-ferromagnet-Ising superconductor may further allow the spin triplet Josephson current to pass [67]. However, constructing such an in-plane junction may require technical ingenuity. In general, heterostructures involving Ising superconductors serve as test-beds not only for further validating Ising pairing but also for achieving exotic properties.

Experiments have shown that the spin-locking effect persists even in bilayer or trilayer of $\mathrm{NbSe}_{2}$ superconductors, for example. It indicates that the local inversion symmetry breaking is sufficient for Ising superconductivity, even though the material itself is globally centrosymmetric. By considering the bilayer case, Liu [27] as well as Nakamura and Yanase [28] predicted that odd-parity superconductivity may occur. This unconventional superconductivity manifests itself either as an inhomogeneous FFLO state or a pair-density-wave state under an in-plane magnetic field. However, it is experimentally quite challenging to detect these exotic states. The current techniques for measuring the FFLO state are often developed for bulk materials. Detecting the pair-density wave 
further requires the understanding of the local density of states in these ultrathin superconductors by using scanning tunneling microscopy (STM).

Carrying out tunneling spectroscopy on Ising superconductors is still under-explored. Many predicted properties such as a "mirage" gap at high energies of the order of $\beta_{S O}$ [38] remain to be verified. It is also of particular interest to obtain the density of states in the regime where the temperature dependent $B_{c 2, \|}$ shows an up-turn, such as in 6-Pb, few-layer stanene, as well as $\mathrm{PdTe}_{2}$. Theories predicted unconventional pairing and possible topological properties in this regime. Furthermore, it is favorable to probe the local density of states. Theoretically, the interplay between Ising superconductors and magnetic impurities were considered by Sharma and Tewari [26] as well as by Zhang et al. [37]. They predicted features that could be identified by STM. Here we list some of the technical challenges of STM investigation on Ising superconductors and possible solutions: 1. Many Ising superconductors are mechanically exfoliated flakes with typical sizes of tens of micrometers. Specially designed markers [68] or optical lens [69] may be necessary to help locate the flake with the tunneling tip. Alternatively, a macro-scale sample, realized for example by MBE, may be favorable; 2 . the ionic liquid gating employed, for example in inducing superconductivity in $\mathrm{MoS}_{2}$ and $\mathrm{WS}_{2}$ Ising superconductors, is incompatible with STM. One may employ alternative gating methods with similarly charge carrier tunability, such as ionic solid/gel gating $[70,71] ; 3$. the exotic phases of Ising superconductors, predicted by theory, often occur at an in-plane magnetic field beyond the Pauli limit. To clearly probe into these phases, it may also be necessary to measure down to low temperature such that $T / T_{c 0}<0.5$. The combination of these requirements make the STM measurement a formidable task. One feasible route may be to realize Ising superconductors with lower $T_{c 0}$ such that $B_{c 2,||}$ stays in the range offered by a commercially available magnet. Reduction of $T_{c 0}$ can be achieved by modulating the carrier density in the existing Ising superconductors.

In order to expand the experimental tools used for studying Ising superconductivity, one may also need to search in the material pool for new systems [39]. There exist several reasons for this proposal. First, many of the existing Ising superconductors require very high magnetic field to induce the predicted exotic states. This is sometimes challenging to be incorporated with other techniques such as STM. Second, nuclear magnetic resonance and thermal capacitance measurements, which are powerful in identifying the FFLO state, may not be easily applied to those atomically thin superconductors. Superlattices, where superconducting sheets are protected by insulating layers, as recently demonstrated by Devarakonda et al. [72] may provide one feasible route. This 
expands the approach to encompass looking for bulk crystals with a stack of Ising superconductors separated by effectively inert layers [73]. Apart from the type-I Ising superconductors that require inversion symmetry breaking, type-II Ising superconductors rely on spin-orbit coupling induced band splittings around high-symmetry points. By using this guiding principle, Wang et al. [23] looked into the database of 2D materials and found a batch of potential candidates for type-II Ising superconductivity, as shown in Fig. 4. This approach was based upon parameterizing the strength of spin-orbit coupling vs Fermi momentum (carrier density). It was taken without significant regard for whether the predicted density could be realized (many materials are in fact semiconductors) or whether a material is actually superconducting. Nevertheless, a number of interesting synthesis paradigms are revealed. While chalcogenides have a relatively rich history of study, the properties of halides are remain relatively poorly explored, especially in the context of thin films, which is the only viable path to making samples sufficiently thin if flakes can not be exfoliated from larger crystals. In general, Ising superconductivity is a vibrant field that has just shown its tip of an iceberg. Experiments led in the beginning, followed by theoretical understandings and predictions for novel physics. Now it is back to the task of experimentalists to probe deeper and wider into this budding field.

\section{ACKNOWLEDGMENTS}

We thank Guangtong Liu, Yong Xu for kindly sharing their data. DZ acknowledges funding provided by the Ministry of Science and Technology of China (2017YFA0302902, 2017YFA0304600); the National Natural Science Foundation of China (grant No. 11922409, 11790311); the Beijing Advanced Innovation Center for Future Chips (ICFC). JF acknowledges funding provided by the Institute for Quantum Information and Matter, an NSF Physics Frontiers Center (NSF Grant PHY-1733907).

[1] H. Rogalla and P. H. Kes, 100 years of superconductivity (Taylor \& Francis, 2011).

[2] P. M. Tedrow and R. Meservey, Phys. Rev. B 8, 5098 (1973).

[3] A. M. Clogston, Phys. Rev. Lett. 9, 266 (1962).

[4] B. S. Chandrasekhar, Appl. Phys. Lett. 1, 7 (1962). 
[5] Y. Liu, Z. Wang, X. Zhang, C. Liu, Y. Liu, Z. Zhou, J. Wang, Q. Wang, Y. Liu, C. Xi, et al., Phys. Rev. X 8, 021002 (2018), URL https://link . aps .org/doi/10.1103/PhysRevX. 8.021002.

[6] Y. Xing, K. Zhao, P. Shan, F. Zheng, Y. Zhang, H. Fu, Y. Liu, M. Tian, C. Xi, H. Liu, et al., Nano letters 17, 6802 (2017).

[7] A. Zhao, Q. Gu, T. J. Haugan, and R. A. Klemm, J. Phys.: Condens. Matter 33, 085802 (2021).

[8] R. A. Klemm, A. Luther, and M. R. Beasley, Phys. Rev. B 12, 877 (1975), URL https://link.aps. org/doi/10.1103/PhysRevB.12.877.

[9] L. P. Gorkov and E. I. Rashba, Phys. Rev. Lett. 87, 037004 (2001).

[10] P. Fulde and R. A. Ferrell, Phys. Rev. 135, A550 (1964), URL https://link.aps.org/doi/10. 1103/PhysRev. 135.A550.

[11] A. Larkin and Y. N. Ovchinnikov, JETP 47, 1136 (1964).

[12] J. M. Lu, O. Zheliuk, I. Leermakers, N. F. Q. Yuan, U. Zeitler, K. T. Law, and J. T. Ye, Science 350, 1353 (2015), ISSN 0036-8075, https://science.sciencemag.org/content/350/6266/1353.full.pdf, URL https://science.sciencemag.org/content/350/6266/1353.

[13] Y. Saito, Y. Nakamura, M. S. Bahramy, Y. Kohama, J. Ye, Y. Kasahara, Y. Nakagawa, M. Onga, M. Tokunaga, T. Nojima, et al., Nature Physics 12, 144 (2016).

[14] X. Xi, Z. Wang, W. Zhao, J.-H. Park, K. T. Law, H. Berger, L. Forró, J. Shan, and K. F. Mak, Nature Physics 12, 139 (2016).

[15] J. Lu, O. Zheliuk, Q. Chen, I. Leermakers, N. E. Hussey, U. Zeitler, and J. Ye, Proceedings of the National Academy of Seiences 115, 3551 (2018), ISSN 0027-8424, https://www.pnas.org/content/115/14/3551.full.pdf, URL https://www . pnas.org/content/115/14/ 3551.

[16] S. C. de la Barrera, M. R. Sinko, D. P. Gopalan, N. Sivadas, K. L. Seyler, K. Watanabe, T. Taniguchi, A. W. Tsen, X. Xu, D. Xiao, et al., Nature communications 9, 1 (2018).

[17] O. Zheliuk, J. M. Lu, Q. H. Chen, A. A. E. Yumin, S. Golightly, and J. T. Ye, Nature Nanotechnology 14, 1123 (2019), URL https://www .nature.com/articles/s41565-019-0564-1.

[18] Y. Tanaka, H. Matsuoka, M. Nakano, Y. Wang, S. Sasakura, K. Kobayashi, and Y. Iwasa, Nano Letters 20, 1725 (2020), pMID: 32013454, https://doi.org/10.1021/acs.nanolett.9b04906, URL https: //doi.org/10.1021/acs.nanolett.9b04906.

[19] L. Bulaevskii, A. Guseinov, and A. Rusinov, Zh. Eksp. Teor. Fiz 71, 2356 (1976).

[20] P. A. Frigeri, D. F. Agterberg, A. Koga, and M. Sigrist, Phys. Rev. Lett. 92, 097001 (2004), URL 
https://link.aps.org/doi/10.1103/PhysRevLett.92.097001.

[21] J. Falson, Y. Xu, M. Liao, Y. Zang, K. Zhu, C. Wang, Z. Zhang, H. Liu, W. Duan, K. He, et al., Science 367, 1454 (2020), ISSN 0036-8075, https://science.sciencemag.org/content/367/6485/1454.full.pdf, URL https://science. sciencemag.org/content/367/6485/1454.

[22] Y. Liu, Y. Xu, J. Sun, C. Liu, Y. Liu, C. Wang, Z. Zhang, K. Gu, Y. Tang, C. Ding, et al., Nano Letters 20, 5728 (2020), pMID: 32584045, https://doi.org/10.1021/acs.nanolett.0c01356, URL https://doi.org/10.1021/acs.nanolett.0c01356.

[23] C. Wang, B. Lian, X. Guo, J. Mao, Z. Zhang, D. Zhang, B.-L. Gu, Y. Xu, and W, Duan, Phys. Rev. Lett. 123, 126402 (2019), URL https://link.aps.org/doi/10 . 1103/PhysRevLett. 123. 126402.

[24] H. Liu, H. Liu, D. Zhang, and X. C. Xie, Phys. Rev. B 102, 174510 (2020).

[25] B. T. Zhou, N. F. Q. Yuan, H.-L. Jiang, and K. T. Law, Phys. Rev. B 93, 180501 (2016), URL https: //link.aps.org/doi/10.1103/PhysRevB.93.180501.

[26] G. Sharma and S. Tewari, Phys. Rev. B 94, 094515 (2016), URL https: //link . aps .org/doi/10. 1103/PhysRevB.94.094515.

[27] C.-X. Liu, Phys. Rev. Lett. 118, 087001 (2017).

[28] Y. Nakamura and Y. Yanase, Phys. Rev. Lett. 96, 054501 (2017).

[29] W.-Y. He, B. T. Zhou, J. J. He, N. F. Yuan, T. Zhang, and K. T. Law, Communications Physics 1, 1 (2018).

[30] P. Lv, Y.-F. Zhou, N.-X. Yang, and Q.-F. Sun, Phys. Rev. B 97, 144501 (2018), URL https : //link. aps.org/doi/10.1103/PhysRevB.97.144501.

[31] D. Möckli and M. Khodas, Phys. Rev. B 98, 144518 (2018), URL https://link .aps.org/doi/ 10.1103/PhysRevB. 98.144518.

[32] D. Möckli and M. Khodas, Phys. Rev. B 99, 180505(R) (2019).

[33] D. Möckli and M. Khodas, Phys. Rev. B 101, 014510 (2020).

[34] D. Wickramaratne, S. Khmelevskyi, D. F. Agterberg, and I. I. Mazin, Phys. Rev. X 10, 041003 (2020).

[35] D. Shaffer, J. Kang, F. J. Burnell, and R. M. Fernades, Phys. Rev. B 101, 224503 (2020).

[36] S. Głodzik and T. Ojanen, New Journal of Physics 22, 013022 (2020), URL https://doi .org/10. $1088 / 1367-2630 / a b 61 d 8$.

[37] Y. Zhang, L. Li, J.-H. Sun, D.-H. Xu, R. Lü, H.-G. Luo, and W.-Q. Chen, Phys. Rev. B 101, 035124 (2020), URL https://link. aps.org/doi/10.1103/PhysRevB. 101.035124.

[38] G. Tang, C. Bruder, and W. Belzig, Phys. Rev. Lett. 126, 237001 (2021), URL https://link . aps . 
org/doi/10.1103/PhysRevLett.126.237001.

[39] X. Zhang and F. Liu, Weyl nodal line induced pairing in ising superconductor and high critical field (2021), 2104.05221.

[40] Y. Saito, T. Nojima, and Y. Iwasa, Nature Reviews Materials 2, 1 (2016).

[41] Y.-H. Lin, J. Nelson, and A. M. Goldman, Physica C 514, 130 (2015).

[42] C. Brun, T. Cren, and D. Roditchev, Supercond. Sci. Technol. 30, 013003 (2017).

[43] A. Kapitulnik, S. A. Kivelson, and B. Spivak, Rev. Mod. Phys. 91, 011002 (2019), URL https: //link.aps.org/doi/10.1103/RevModPhys.91.011002.

[44] B. Sacepe, M. Feigelman, and T. M. Klapwijk, Nat. Phys. 16, 734 (2020).

[45] M. Kim, Y. Kozuka, C. Bell, Y. Hikita, and H. Y. Hwang, Phys. Rev. B 86, 085121 (2012), URL https://link.aps.org/doi/10.1103/PhysRevB.86.085121.

[46] M. Ben Shalom, M. Sachs, D. Rakhmilevitch, A. Palevski, and Y. Dagan, Phys. Rev. Lett. 104, 126802 (2010).

[47] T. Sekihara, R. Masutomi, and T. Okamoto, Phys. Rev. Lett. 111, 057005 (2013), URL https:// link.aps.org/doi/10.1103/PhysRevLett.111.057005.

[48] H. Nam, H. Chen, T. Liu, J. Kim, C. Zhang, J. Yong, T. R. Lemberger, P. A. Kratz, J. R. Kirtley, K. Moler, et al., Proceedings of the National Academy of Sciences 113, 10513 (2016), ISSN 0027-8424, https://www.pnas.org/content/113/38/10513.full.pdf, URL https: //www . pnas .org/ content/113/38/10513.

[49] S. Ilic, J. S. Meyer, and M. Houzet, Phys. Rev. Lett. 119, 117001 (2017), URL https://link . aps. org/doi/10.1103/PhysRevLett.119/117001.

[50] V. Fatemi, S. Wu, Y. Cao, L. Bretheau, Q. D. Gibson, K. Watanabe, T. Taniguchi, R. J. Cava, and P. Jarillo-Herrero, Science 362, 926 (2018), ISSN 0036-8075, https://science.sciencemag.org/content/362/6417/926.full.pdf, URL https://science.sciencemag. org/content/362/6417/926.

[51] E. Sajadi, T. Palomaki, Z. Fei, W. Zhao, P. Bement, C. Olsen, S. Luescher, X. Xu, J. A. Folk, and D. H. Cobden, Science 362, 922 (2018), ISSN 0036-8075, https://science.sciencemag.org/content/362/6417/922.full.pdf, URL https://science.sciencemag. org/content/362/6417/922.

[52] J. Cui, P. Li, J. Zhou, W.-Y. He, X. Huang, J. Yi, J. Fan, Z. Ji, X. Jing, F. Qu, et al., Nature communications 10, 1 (2019). 
[53] D. A. Rhodes, A. Jindal, N. F. Yuan, Y. Jung, A. Antony, H. Wang, B. Kim, Y.-c. Chiu, T. Taniguchi, K. Watanabe, et al., Nano Letters (2021).

[54] Y.-M. Xie, B. T. Zhou, and K. T. Law, Phys. Rev. Lett. 125, 107001 (2020).

[55] M. Liao, Y. Zang, Z. Guan, H. Li, Y. Gong, K. Zhu, X.-P. Hu, D. Zhang, Y. Xu, Y.-Y. Wang, et al., Nature Physics 14, 344 (2018).

[56] J. Peng, Y. Liu, X. Luo, J. Wu, Y. Lin, Y. Guo, J. Zhao, X. Wu, C. Wu, and Y. Xie, Advanced Materials 31, 1900568 (2019), URL https://onlinelibrary.wiley.com/doi/abs/10.1002/ adma. 201900568 .

[57] E. Sohn, X. Xi, W.-Y. He, S. Jiang, Z. Wang, K. Kang, J.-H. Park, H. Berger, L. Forró, K. T. Law, et al., Nature materials 17, 504 (2018).

[58] T. Dvir, F. Massee, L. Attias, M. Khodas, M. Aprili, C. H. Quay, and H. Steinberg, Nature communications 9, 1 (2018).

[59] D. Costanzo, H. Zhang, B. A. Reddy, H. Berger, and A. F. Morpurgo, Nature nanotechnology 13, 483 (2018).

[60] K. Kang, S. Jiang, H. Berger, K. Watanabe, T. Taniguchi, L.Forro, J. Shan, and K. F. Mak, Giant anisotropic magnetoresistance in ising superconductor-magnetic insulator tunnel junctions (2021), 2101.01327.

[61] A. Hamill, B. Heischmidt, E. Sohn, D. Shaffer, K.-T. Tsai, X. Zhang, X. Xi, A. Suslov, H. Berger, L. Forro, et al., Nat. Phys. pp. 10.1038/s41567-021-01219-x (2021).

[62] N. F. Q. Yuan, K. F. Mak, and K. T. Law, Phys. Rev. Lett. 113, 097001 (2014), URL https: //link. aps.org/doi/10.1103/PhysRevLett/113.097001.

[63] M. Haim, D. Möckli, and M. Khodas, Phys. Rev. B 102, 214513 (2020), URL https://link . aps . org/doi/10.1103/PhysRevB. 102.214513.

[64] M. Kuzmanovic, T, Dvir, D. LeBoeuf, S. Ilic, D. Möckli, M. Haim, S. Kraemer, M. Khodas, M. Houzet, J. S. Meyer, et al., Tunneling spectroscopy of few-monolayer nbse 2 in high mangetic field: Ising protection and triplet superconductivity (2021), 2104.00328.

[65] V. Mourik, K. Zuo, S. M. Frolov, S. R. Plissard, E. P. A. M. Bakkers, and L. P. Kouwenhoven, Science 336, 1003 (2012), ISSN 0036-8075, https://science.sciencemag.org/content/336/6084/1003.full.pdf, URL https://science. sciencemag . org/content/336/6084/1003.

[66] Y. Xie, B. T. Zhou, T. K. Ng, and K. T. Law, Phys. Rev. Research 2, 013026 (2020), URL https: //link.aps.org/doi/10.1103/PhysRevResearch.2.013026. 
[67] Q. Cheng and Q.-F. Sun, Phys. Rev. B 99, 184507 (2019), URL https://link.aps.org/doi/10. 1103/PhysRevB.99.184507.

[68] G. Li, A. Luican, and E. Y. Andrei, Rev. Sci. Instrum. 82, 073701 (2011).

[69] V. Geringer, M. Liebmann, T. Echtermeyer, S. Runte, M. Schmidt, R. Ruckamp, M. C. Lemme, and M. Morgenstern, Phys. Rev. Lett. 102, 076102 (2009).

[70] M. Liao, Y. Zhu, J. Zhang, R. Zhong, J. Schneeloch, G. Gu, K. Jiang, D. Zhang, X. Ma, and Q.-K. Xue, Nano Lett. 18, 5660 (2018).

[71] R. Yin, L. Ma, Z. Wang, C. Ma, X. Chen, and B. Wang, ACS Nano 14, 7513 (2020).

[72] A. Devarakonda, H. Inoue, S. Fang, C. Ozsoy-Keskinbora, T. Suzuki, M. Kriener, L. Fu, E. Kaxiras, D. C. Bell, and J. G. Checkelsky, Science 370, 231 (2020), ISSN 0036-8075, https://science.sciencemag.org/content/370/6513/231.full.pdf, URL https://science.sciencemag. org/content/370/6513/231.

[73] H. Zhang, A. Rousuli, S. Shen, K. Zhang, C. Wang, L. Luo, J. Wang, Y. Wu, Y. Xu, W. Duan, et al., Science Bulletin 65, 188 (2020), ISSN 2095-9273, URL https : / www . sciencedirect . com/ science/article/pii/S2095927319306656.

[74] J. Zeng, E. Liu, Y. Fu, Z. Chen, C. Pan, C. Wang, M. Wang, Y. Wang, K. Xu, S. Cai, et al., Nano Letters 18, 1410 (2018), pMID: 29385803, https://doi.org/10.1021/acs.nanolett.7b05157, URL https://doi.org/10.1021/acs.nanolett.7b05157. 


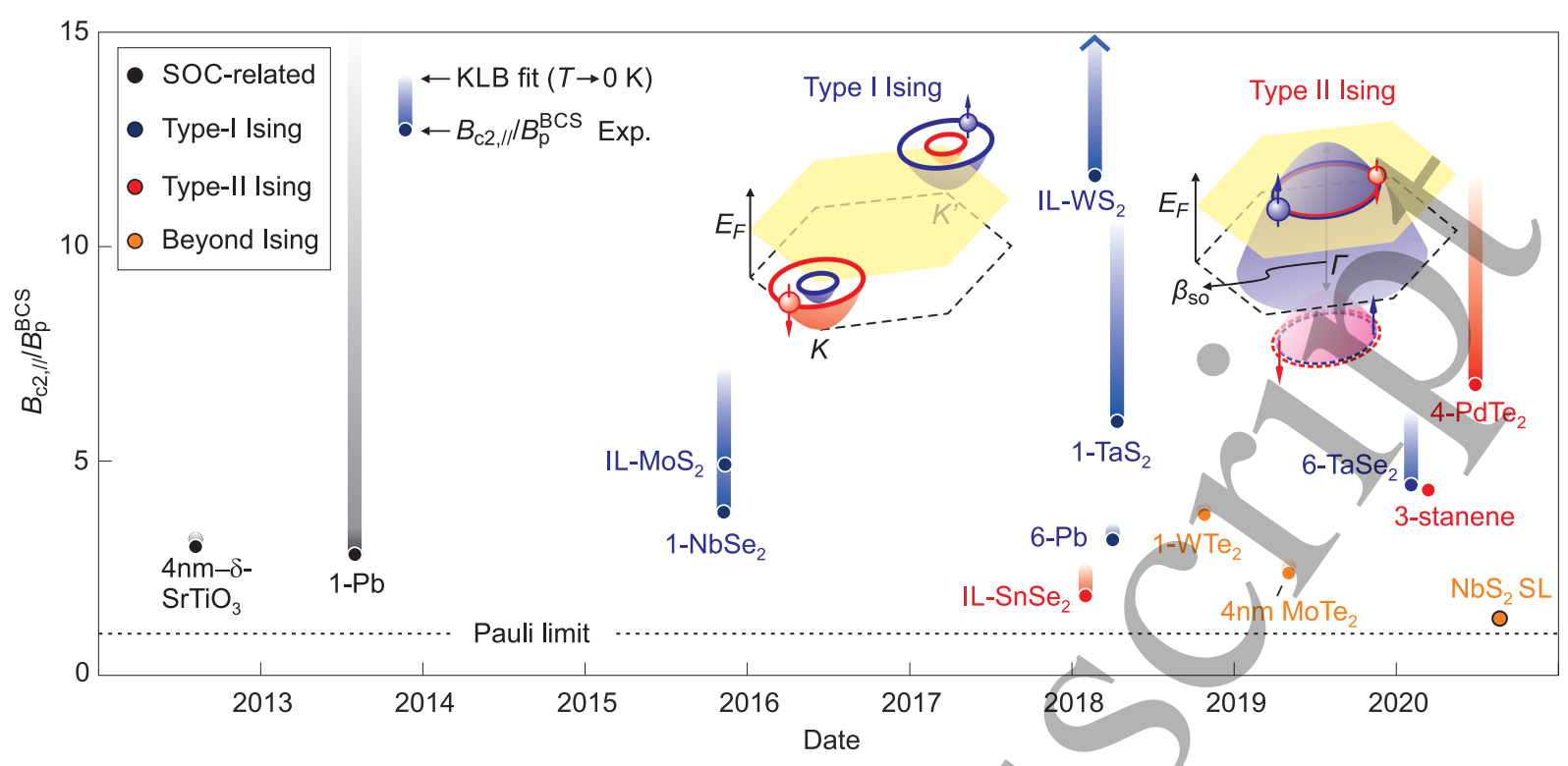

FIG. 1. Two-dimensional superconductors with enhanced in-plane upper critical fields. Black: spinorbit coupling (SOC) related systems [45, 47], Blue: Type-I Ising superconducting materials [5, 12, 1416, 18, 74]. Red: Type-II Ising superconducting materials [21, 22]. Orange: "beyond" Ising-type superconductivity $[50-52,72]$. The solid symbols present the experimentally measured $B_{c 2,||} / B_{p}^{B C S}$ ratio in each reference, which is often limited by the magnet field available. The upper bound of the faded bar indicates the extrapolated $B_{c 2, \|} / B_{p}^{B C S}$ ratio at zero temperature according to the KLB fit to the temperature dependent upper critical field (shown for example/in Fig. 2). For triple layer stanene (3-stanene) and $\mathrm{NbS}_{2}$ in a superlattice $\left(\mathrm{NbS}_{2}-\mathrm{SL}\right)$, the measurements were carried down to temperature close to absolute zero such that the extrapolation is no longer necessary. Note that the value for $\mathrm{WS}_{2}$ exceeds the vertical axis limit of 15 . Here, "IL" corresponds to ionic liquid gated samples and the number preceding the chemical tag indicates the number of monolayers or total thickness of the device. Dashed line marks the Pauli limit. Schematic drawing as insets illustrate the band structure with spin-splitting for two types of Ising superconductivity. For type-I Ising, only one pair of bands around the $\mathrm{K} / \mathrm{K}^{\prime}$ points is shown. 

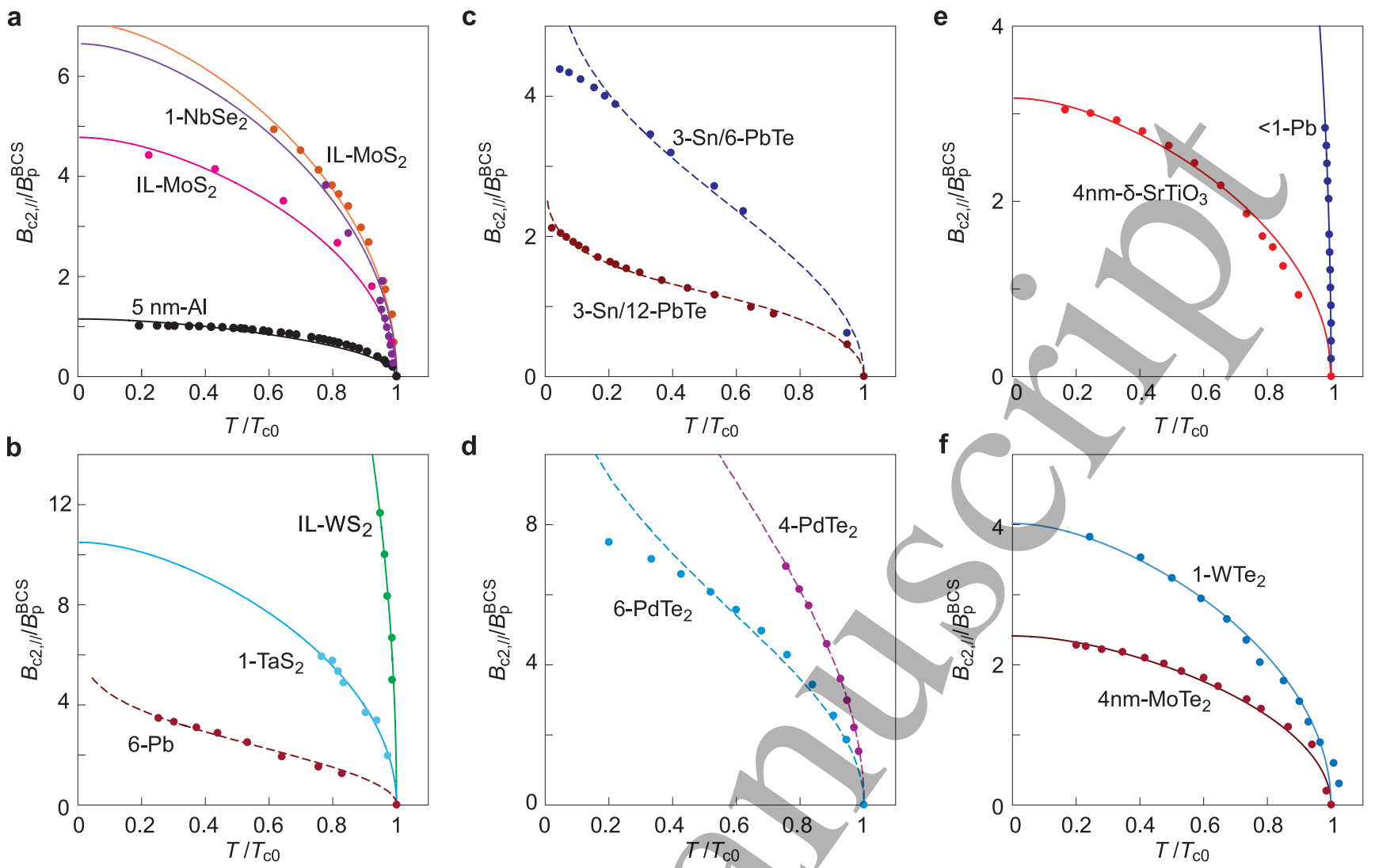

FIG. 2. In-plane upper critical fields of selected two-dimensional superconductors. Circles are data points digitized from the corresponding references (a: Refs. [2, 12-14]; b: Refs. [15, 16, 27]; c: Ref. [21]; d: Ref. [22]; e: Refs. [45, 47]; f: Refs. [50, 52]). Panels a and b include data from several type-I Ising superconductors. Data points for the thin aluminum are shown for comparison. Panels $\mathrm{c}$ and d include data from type-II Ising superconductors. Panels e and f show data from other 2D superconductors in which the enhanced $B_{c 2, \|}$ stems from strong spin-orbit coupling effect. Solid curves are theoretical fits by using the KLB formula [Eq. (5)]. Dashed curves are fits by using the formula for Ising superconductors [Eq. (6)]. In panels $\mathrm{c}$ and $\mathrm{d}$, deviation between the dashed curve and the data points at low temperatures indicates that the additional Rashba effect need to be taken into account. 


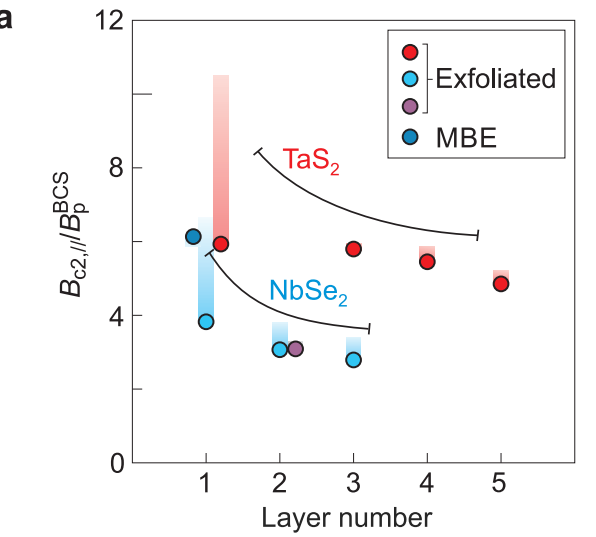

b

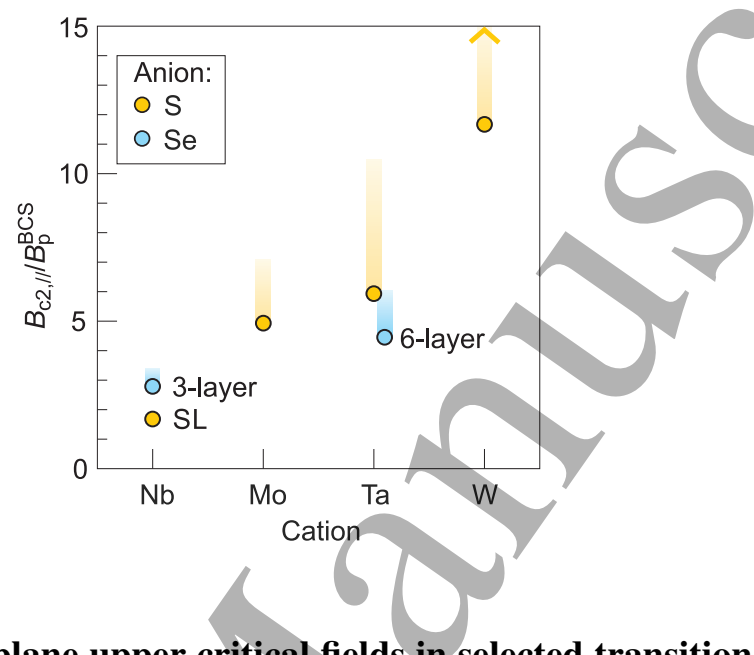

FIG. 3. Enhancement of the in-plane upper critical fields in selected transition metal dichalcogenide superconductors. Comparison of $B_{c 2, \|} / B_{p}^{B C S}$ as a a function of layer number for $\mathrm{TaS}_{2}[16]$ and $\mathrm{NbSe}_{2}[6$, $14,16]$, and $\mathbf{b}$ a function of cation $[12-16,18,72]$. The extrapolated $B_{c 2, \|} / B_{p}^{B C S}$ value for $\mathrm{WS}_{2}$ exceeds the vertical axis limit of 15 . The value for $\mathrm{NbS}_{2}$ is taken from the measurement on a superlattice (SL) consisting of $\mathrm{NbS}_{2}$ layers [72]. The relatively small value here may also be influenced by the strong Rashba effect present in the system. For selenide compounds, we compare a six-layer $\mathrm{TaSe}_{2}$ in the $3 \mathrm{R}$-phase-the thinnest one reported with a trilayer of $2 \mathrm{H}-\mathrm{NbSe}_{2}$, because data from thicker $\mathrm{NbSe}_{2}$ is not available. 


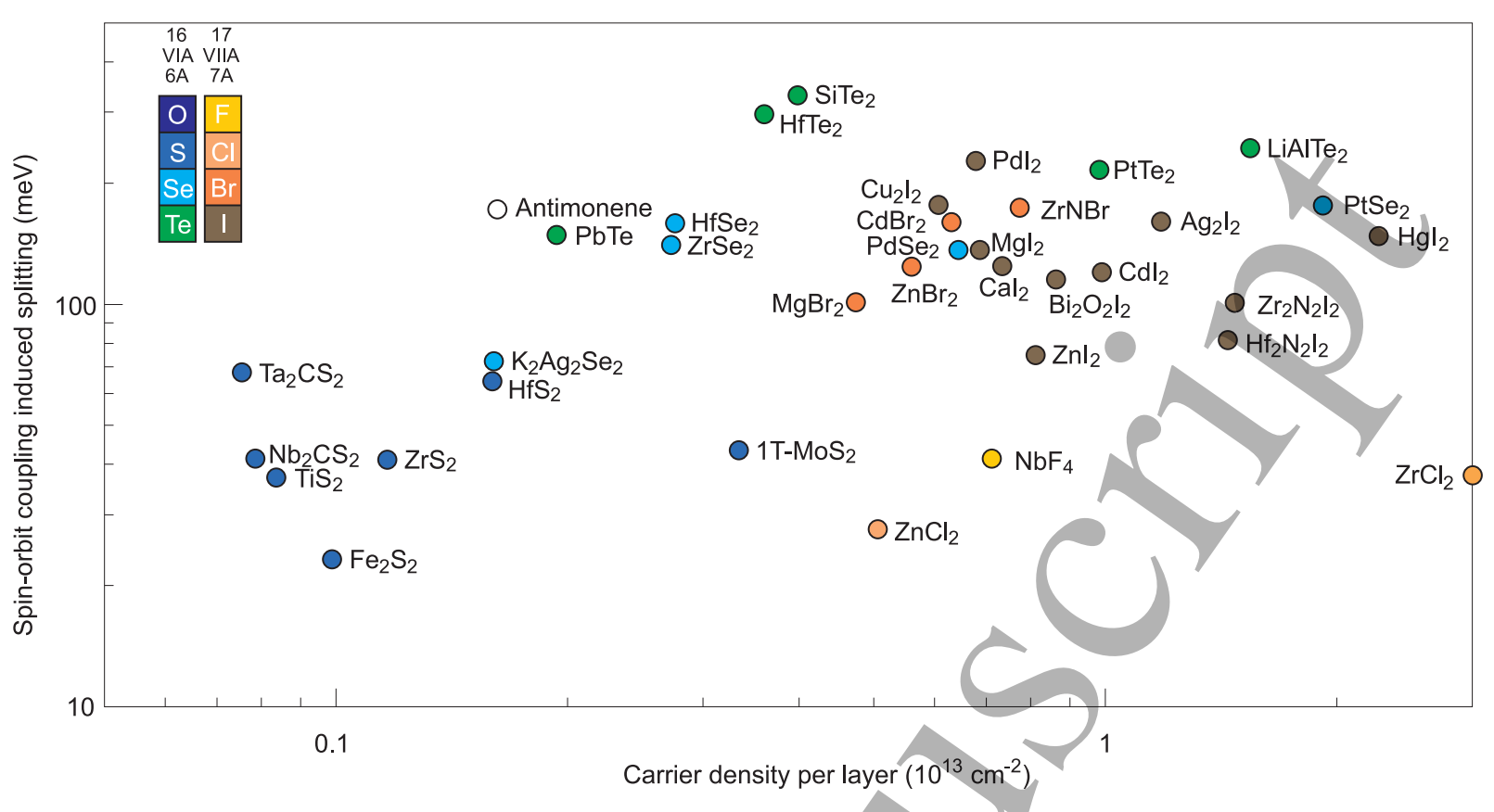

FIG. 4. Predicted Type-II Ising superconductors. Material platforms color coded according to anion type as a function of carrier density per lay and spin-orbit coupling induced splitting. Data points are from Ref. 23. It was proposed that the Fermi level should be tuned such that the carrier density stays below the listed values to possess pronounced enhancement. Note that $\mathrm{ZrCl}_{2}$ possesses large splitting across the whole momentum space. 